\title{
Comparison of Biopsy Results and Imaging Methods, in the Diagnosis of Breast Cancer
}

\author{
Mehmet Ali Eryılmaz $^{1 *}$, Özgür Öner ${ }^{2}$, Ahmet Okuş $^{1}$, Ömer Karahan $^{1}$, Said Bodur $^{3}$, \\ Serden Ay ${ }^{1}$, Seher Civcik ${ }^{4}$ \\ ${ }^{1}$ General Surgery Clinic, Konya Training and Research Hospital, Konya, Turkey \\ ${ }^{2}$ Radyology Clinic, Konya Training and Research Hospital, Konya, Turkey \\ ${ }^{3}$ Department of Public Health, Meram Faculty of Medicine, Selçuk University, Konya, Turkey \\ ${ }^{4}$ Konya Early Diagnosis of Cancer-Screening and Education Center, \\ Kanser Erken Teşhis-Tarama ve Eğitim Merkezi (KETEM), Konya, Turkey \\ Email: *mali_eryilmaz@hotmail.com
}

Received July 13, 2012; revised August 14, 2012; accepted September 2, 2012

\begin{abstract}
Purpose: In this study, we aimed at comparing the sensitivity and selectivity rates of Ultrasonography (USG), Mamography (MG) and Magnetic Resonance (MR), based on the biopsy results of breast mass lesions. Materials and Methods: Between January 2009 and December 2010 in Konya Training and Research Hospital the biopsy results and reports of imaging tecniques such as USG, MG and MR, were obtained from the hospital automation. The sensitivity and selectivity of the USG, MG and MR were calculated. Results: The avarage age of the 112 patients included in this study is $49 \pm 10(23-71) .27(24 \%)$ of the patients were found to have breast cancer after the histopathologic examination. USG was used with the $95(94 \%)$ of the patients and $17(18 \%)$ of the patients were diagnosed to have cancer. MG was used with the $75(67 \%)$ of the patients and $15(20 \%)$ of the patients were diagnosed to have cancer, and MR was used with the $112(100 \%)$ of the patients and $25(22 \%)$ of the patients were diagnosed to have cancer. In the pathologic diagnosis of cancer, the sensitivity of USG was found $85 \%$, the sensitivity of MG was found $89 \%$, and the sensitivity of MR was found $92 \%$. The selectivity of these tecniques were $58 \%, 87 \%$ and $57 \%$ respectively. Conclusion: MR and MG have similar sensitivity rates in the diagnosis of breast cancer. Breast MR is an appropriate imaging tecnique that can be used in suitable indications in addition to USG and MG.
\end{abstract}

Keywords: Breast Cancer; Magnetic Resonance; Mamography; Ultrasonography

\section{Introduction}

Breast cancer is still at the top of list of morbidity and mortality besides being one of the most common type of cancer among women both in the World and in our country [1]. MG is still the basic method for scanning and the determining the breast lesions with its $90 \%$ sensitivity rate [2-4]. Some researchs reveal that MG cannot determine the breast cancer with a $10 \%-30 \%$ rate [5]. USG is the first method to be used when mamography is insufficient with sclerosing breast tissue [6]. USG is more sensitive with women who are under the age of 40 and who have dens breast tissue [7]. USG and MG is insufficient in evaluating the behavioral characteristics of the determined lesions, determining the focus occurance of the multifocalmulticentric (mf-mc) pre-breast conserving surgery, differentiating between the residue lesion and the granulation tissue and the post treatment monitoring and in such

${ }^{*}$ Corresponding author. situations MR examination is required to solve the problem in routine applicaitons $[2,8,9]$. With the use of contrast agent in MR, it is easier to differentiate between the belign and malign lesions.

Procedures that are suitable to age or the characteristics of the mass and efficient examinations increases the determination rate of the cancer, whereas redundant tests and procedures decreases this rate [10].

This research aims at comparing the sensitivity and selectivity rates USG, MG and MR based on the biosy results of the breast mass lesions.

\section{Materials and Methods}

\subsection{Patients}

The files that belong to the patients who consulted to Konya Training and Research Hospital with a breast complaint and were applied breast MR were studied retrocpectively. It was determined that, every patient that consulted to 
General Surgery Clinic with a breast complaint was first applied Clinical Breast Examination (CBE). After that, bilaretal Breast USG examination was done to patients under the age of 40 and bilateral MG examination was done to patients over the age of 40 . USG and MG reports were arranged according to BIRADS classification. Breast MR was done to patients who were found to have BIRADS-0, BIRADS 4 and 5 in USG and MG, who has a breast cancer history in the family and dense breast tissue. Moreover, MR was done to patients who were diagnosed to have breast cancer and planned to be applied breast concerving surgery, in order to determine the multicentric-multifocal focuses and evaluate the pectoral phaisa or breast-wall invasion.

Breast MR was also done to patients who have lesions suspected to have breast cancer defined as BIRADS-3 in USG and MG in order to define the characteristics of the lesion, in the cases which were found to be suspected in the CBE but no tumors were deifned mamographically and sonographically and the patients who were found to have structural distortion in the mamographies but not in sonographies.

BIRADS classification was used to define the lesions found the breast MR. Tru-cut biopsy with USG was applied to patients whose breast lesions were suspected to be BIRADS-4 or 5 in at least one of USG, MG and MR. The patients who were suspected to have microcalcifications suspected to malign in MG were send to an epicenter to be applied steraotaxic marked biopsy and those patients were not included in the study.

\subsection{Breast Ultrasonography (USG)}

USG examination was applied to the patients in the supine position with Siemens Antares device and lineer prob with a 5 - $15 \mathrm{MHz}$ wide frequency. BIRADS classification was used defining the lesions found in the USG.

\subsection{Mammography (MG)}

Mammographical examination was done to both breasts in two standard positions (MLO and CC). Visions acquired using Cintek Glory MG device were transformed into digital visions using Agfa CR85-X CR(computerized radiology) system. BIRADS classification was used defining the lesions determined in the MG.

\subsection{Breast Magnetic Resonance (MR) Examination}

Breast MR was done after the USG and/or MG examinations with the request of radiologist or general surgery specialist. In the cases that are not urgent, MR examinations were done in the 2 nd week of the menstrual cycle.
Dynamic enhanced Breast MR examination were done in prone position using 1.5 Tesla Siemens Avanto MR device and 4 channel breast coil. After the localizer visions were acquired, T1-weighted spin eco fat supressed free coronal sequence (TR: 313, TE: 4.5, FOV $350 \mathrm{~mm}$, Matrix $256 \times 230$, Section Thickness $3 \mathrm{~mm}$ ), T2-weighted fast spin eco fat supressed free axial sequence (TR:9710, TE: 190, FOV: 350 mm, Matrix $384 \times 288$, Section Thickness $3 \mathrm{~mm}$ ), T2-weighted TRIM axial sequence sekans (TR: 2770, TE: 68, FOV: $350 \mathrm{~mm}$, Matrix $320 \times 272$, Section Thickness $3 \mathrm{~mm}$ ) and T1-weighted 3D gradient eco dynamic sequnece in the axial plan (TR: 4.43, TE: 1.35, FOV: $330 \mathrm{~mm}$, Matrix $448 \times 313$, Section Thickness $1 \mathrm{~mm}$ ) were acquired. In the dynamic examination, unenhanced fat supressed free T1-weighted base vision was acquired first. Then, $0.1 \mathrm{mmol}$ gadolinium-DTPA/ $\mathrm{kg}$ was given as intravenous and enhanced sequence was repeated 5 times consequetively. Substraction visions were acquired excluding the base visions from the enhanced visions. Signaltime curve was formed using the substraction visions. The breast masses were evaluated taking their morphological and contrast agent involvement characteristics into consideration. The lesions smaller than 5 $\mathrm{mm}$ were evaluated as focus, whereas lesions bigger than 5 mm were evaluated as mass lesion. The shape of the mass (round, oval, lobular or irregular), the contours of the mass (smooth, irregular, spicule) and contrast involvment characteristics of the mass (homogenous, heterogenous, annular or central) were used evaluating the lesion. Evaluating the non-massive contrast involvment zones, dispersion (focal, multifocal, lineer, ductal, segmental or diffuse), contrast involvement characteristics (homogenous, heterogenous, punctual, nodular interreptus or reticular) and either they are symmetrical or not were taken into consideration. In the kinetic evaluation, contrasting rate (slow, medium or fast), and contrasting pattern (increasing in due course, forming plateaus or forming swift contrast loss) were used acquiring time-signal curve. Defining the lesions determined in MR, BIRADS classification was used.

\subsection{Biopsy Procedure}

For pathological examination, at least 3 pieces of sample were taken with a full-automatic tru-cut needle with the angled-prob of the USG, from the patients whose mass lesions were found to be BIRADS 4 - 5 in the USG, MG and MR. These were sent to pathology laboratory in the $10 \%$ formol with the material biopsy container.

All the USG, MG and MR monitoring reports and pathology results of all the patients were acquired from the hospital automation. The sensitivity and selectivity of USG, MG and MR examinations of these patients in the pathological diagnosis were computed. 


\subsection{Statistics}

Sensitivity, Specifitiy, Positive Predictive value, Negative Predictive value, Total Truth values were used. $P$ value was calculated using SPSS (SPSS Inc.16 2009, Chicago, IL, USA) statistics software. $\mathrm{P}<0.05$ was accepted as significant.

\section{Results}

The avarage age of the 112 patients included in this study is $49 \pm 10$ (23 - 71). In the histopathological examinations of the biopsies taken with the suspect of cancer, 27 (24\%) of the patients were found to have breast cancer. $21(77 \%)$ of these have invasive ductal carcinoma (Table 1). USG was applied to 95 (94\%) of the patients and $17(18 \%)$ of these were found to have cancer. MG was applied to 75 $(67 \%)$ of the patients and $15(20 \%)$ of these were found to have cancer. MR was applied to $112(100 \%)$ of the patients and $25(22 \%)$ of these were found to have cancer. The sensitivity of the USG is $85 \%$, MG is $94 \%$ and MR is $92 \%$ in the pathological cancer diagnosis. The selectivity rates of these respectively are; $58 \%, 83 \%$ and $57 \%$ (Table 2).

USG examination was done to 95 (94\%) of the 112 patients. MR was done to 2 breasts defined as BIRADS- 0 additionally, evaluated as BIRADS-4 and the biopsy results showed that these were benign. MR was applied and biopsy was taken from $46(41 \%)$ of the breasts defined as BIRADS- 4 and 5 additionally and 16 (14\%) of these were found to have cancer (Table 3).

MG was done to $75(67 \%)$ of the 112 patients. MR was done to $48(42 \%)$ of the patients who were evaluated as BIRADS-0 additionally. $19(40 \%)$ of these were evaluated as BIRADS-4 and $9(18 \%)$ of them were evaluated as BIRADS-5 in the MR and biopsies were taken from these patients (Figure 1). 10 (21\%) of these patients were found to have cancer (Table 3).

Both MG and USG was done to a single patient and evaluated as BIRADS-5 in USG and BIRADS-2 in MG. The same patient was evaluated as BIRADS- 2 in MR and with the biopsy, she was diagnosed to have cancer. 1 (4\%) of the 27 cancer diagnoses were reached at the correct diagnosis in the USG evaluation.

MR was done to 5 patients additionally who were evaluated as BIRADS-4 in MG and 3 of these were evaluated as BIRADS-2 (Figure 2). Biopsy was taken from these patients as MG results were suspected to be malign, however the result was benign. The other 2 of these were evaluated as BIRADS-5 in MR and biopsy was taken from these patients and these patients were diagnosed to have cancer. With the cases diagnosed as BIRADS-4 in MG, Breast MR examination was found to be significant in diagnosing the cancer $(\mathrm{P}<0.05)$.

MR was done to 3 patients who were defined as BIRADS-5 in MG, in order to examine the occurance of multifocal-multicentric lesions and 2 breasts were evaluated as BIRADS-5 and unifocal cancer was diagnosed after the biopsy an done of these patients were found to have multifocality and with the biopsy, she was diagnosed to have multifocal cancer.

Breast MR was applied to 112 patients. Biopsy was taken from 38 breasts that were evaluated as BIRADS-4 and 5 of these were diagnosed to have breast cancer. Biopsy was taken from the 23 breasts evaluated as BIRADS- 5 and 20 of these breasts were diagnosed to have cancer. Two breasts were defined as benign with MR and with the biopsy these were evaluated as cancer. Two of these cancers were determined with only USG and the other was determined with MG (ductal carcinoma insitu) (Table 4). After the breast reduction surgery, two breasts were evaluated as BIRADS-5 with USG and as BIRADS-0 with MG. The MR results of these patients were evaluated as BIRADS-2 and the biopsy results were reported as benign.

\section{Discussion}

Breast Cancer is one of the most important reasons of the deaths of cancer among women [11]. To decrase the death rate of the breast cancer, it is important to diagnose at an

Table 1. Histopathological distribution of the breast cancers.

\begin{tabular}{ccc}
\hline Histopathology & $\mathrm{N}$ (number) & $\%$ \\
\hline $\begin{array}{c}\text { Invasive ductal carcinoma } \\
\text { Invasive ductal carcinoma + Invasive } \\
\text { lobular carcinoma }\end{array}$ & 21 & 77.8 \\
Invasive lobular carcinoma & 3 & 11.1 \\
Medullar carcinoma & 1 & 3.7 \\
DCIS & 1 & 3.7 \\
Total & 1 & 3.7 \\
\hline
\end{tabular}

Table 2. The sensitivity and selectivity rates of the monitoring methods in the diagnosis of breast cancer.

\begin{tabular}{cccccc}
\hline İmaging methods & Sensitivity (\%) & Specifity (\%) & Positive predictive value (\%) & Negative predictive value (\%) & Truth value (\%) \\
\hline USG & 85.0 & 58.3 & 36.2 & 93.3 & 64.1 \\
MG & 94.4 & 83.3 & 85.0 & 93.8 & 88.9 \\
MR & 92.6 & 56.8 & 41.7 & 95.8 & 65.7 \\
\hline
\end{tabular}


Table 3. Consistency of the BIRADS classification with the diagnosis of breast cancer.

\begin{tabular}{|c|c|c|c|c|c|c|c|c|c|c|c|c|}
\hline \multirow[b]{2}{*}{$\begin{array}{l}\text { İmaging } \\
\text { methods }\end{array}$} & \multicolumn{3}{|c|}{ BIRADS-0 } & \multicolumn{3}{|c|}{ BIRADS-1-2-3 } & \multicolumn{3}{|c|}{ BIRADS-4 } & \multicolumn{3}{|c|}{ BIRADS-5 } \\
\hline & $\begin{array}{c}\text { Number } \\
\text { (n) }\end{array}$ & $\mathrm{Ca}$ & $\%$ & $\begin{array}{c}\text { Number } \\
\text { (n) }\end{array}$ & $\mathrm{Ca}$ & $\%$ & $\begin{array}{c}\text { Number } \\
\text { (n) }\end{array}$ & $\mathrm{Ca}$ & $\%$ & $\begin{array}{c}\text { Number } \\
\text { (n) }\end{array}$ & $\mathrm{Ca}$ & $\%$ \\
\hline USG & 2 & 0 & 0.0 & 47 & 3 & 6.9 & 34 & 4 & 11.7 & 12 & 10 & 83.3 \\
\hline MG & 48 & 10 & 20.8 & 19 & 2 & 10.5 & 5 & 2 & 40.0 & 3 & 3 & 100.0 \\
\hline
\end{tabular}

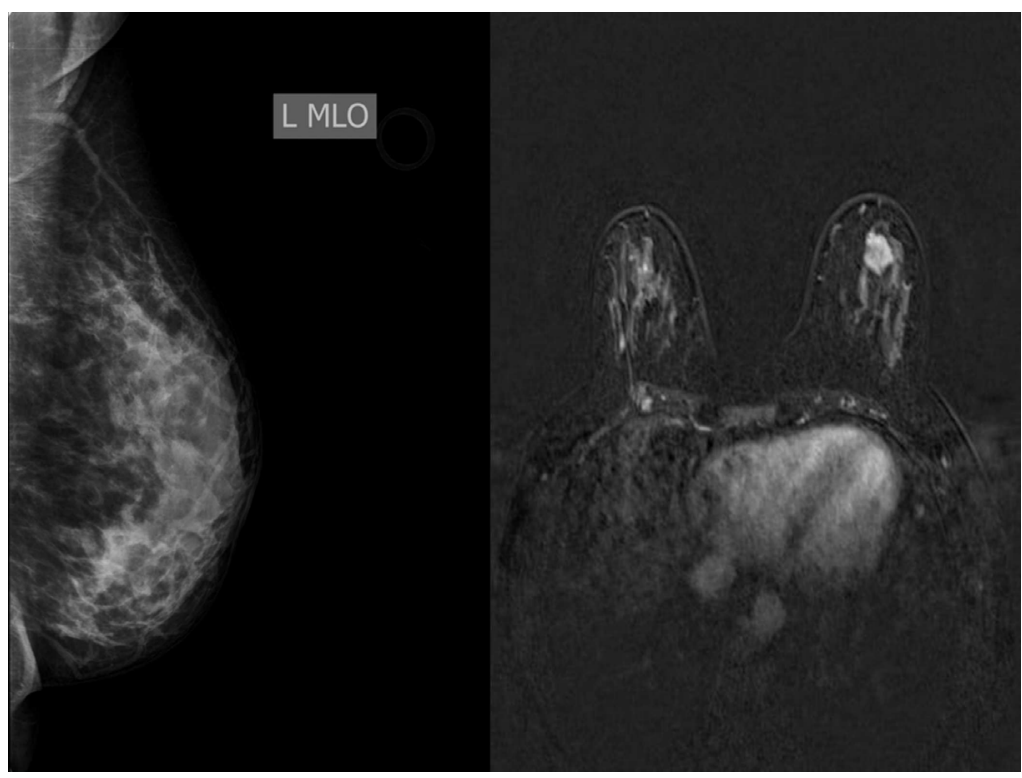

Figure 1. Glandular tissues and suspicious nodular opacities that cannot be qualified as net superimposition are present in mammography of left breast subareolar area. In the US and MR examination, $21 \times 19 \mathrm{~mm}$ sized mass lesion with a malign outlook in the left breast lower quadrant and numerous nodular lesions smaller than $5 \mathbf{~ m m}$ than can be compliant with satellite lesions in the left breast were detected.

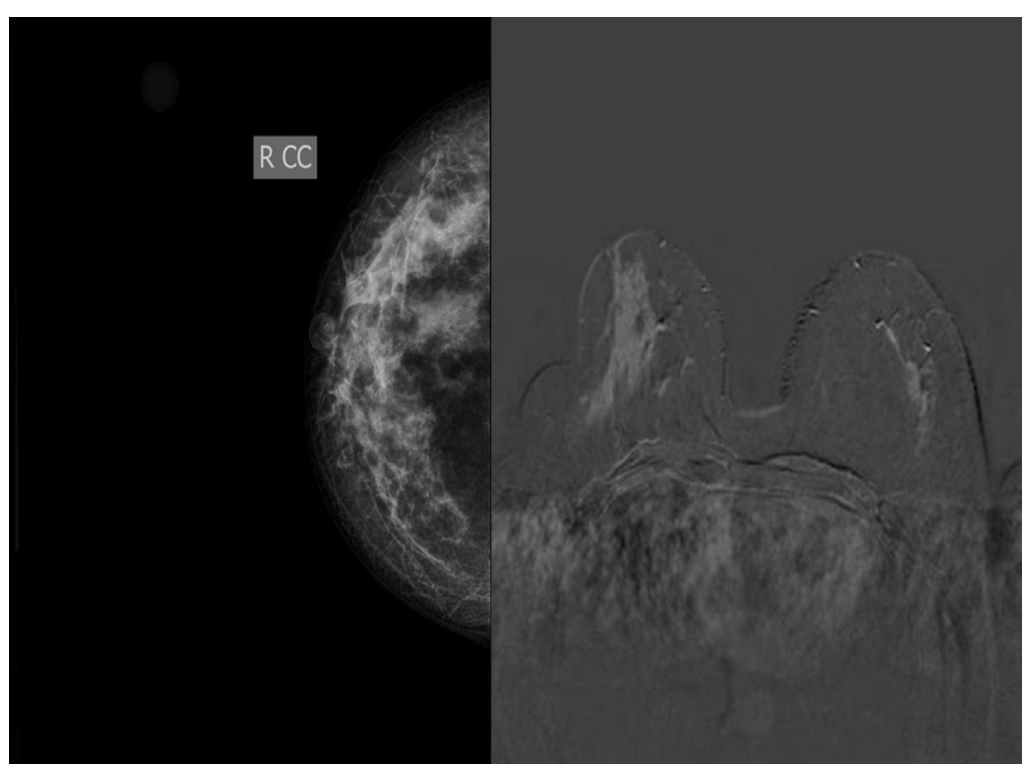

Figure $2.2 \mathrm{~cm}$ diameters of asymmetric density is observed in the CC graphy outer quadrant of left breast in the mammography. With the MR, no pathologies but a couple of millimetric cysts were observed. With the US, the result of the biopsy got from the heterogenous area which cannot be lined off precisely in the periareolar area of left breast revealed situ carcinoma (2nd degree). 
Table 4. The number of the lesions that were found to have cancer with biopsy but cannot be monitored with monitoring methods $(n=27)$.

\begin{tabular}{ccc}
\hline İmaging methods & $\mathrm{n}$ & $\%$ \\
\hline USG & 3 & 11.1 \\
MG & 2 & 7.4 \\
MR & 2 & 7.4 \\
USG + MG & 2 & 7.4 \\
MG + MR & 1 & 3.7 \\
USG + MR & 1 & 3.7 \\
USG + MG + MR & 0 & 0.0 \\
\hline
\end{tabular}

early stage and correctly. Mammography is a monitoring method that decreases the death rate of the breast cancer [12]. Death rate of the breast cancer from the age of 40 has decreased at a $31 \%$ rate with the breast cancer scanning with mammography [13]. It is adviced for every woman to have a CBE with mammography once a year $[14,15]$. Mammography has a high sensitivity rate in routine scans and patients with breast symptoms. The use of mammography with USG increases the sensitivity rate and changes the follow-up procedures [16]. None of the methods has the adequate sensitivity, specifity and diagnostically correctness rate in the diagnosis of the breast lesions. Therefore, it is not true to use one single method in the diagnosis of the breast lesions and combining a couple of methods together increases the diagnostical correctness rate. In this study, diagnosing the patients suspected to have cancer in the anamnesis and physical examinations, when at one of CBE, USG, MG and MR methods indicated the occurance of cancer, biopsy was applied with USG.

About $20 \%$ of the breast cancers are observed under the age of 40 , whereas $30 \%$ of these are observed under the age of 50. To minimise the number of the redundant tests, the age and the characteristics of the breasts should be taken into consideration. Because of the dense characteristics of the breast tissue, not MG but USG is more significant with the women patients under age of 40 . MG is more significant with the women over the age of 40 and it is suggested to be applied once a year $[10,12]$. In this study, $18 \%$ of the patients diagnosed to have cancer are under the age of 40 and the $33 \%$ of these are under the age 50 .

Diagnostical MG has a $89 \%$ rate sensitivity in the determining the breast cancer and the masses that can be felt with hand is normal in the $9 \%-20 \%$ of the breast cancers [10]. In this study, a mass lesion that can be felt with hand was found in 1 (4\%) of the patients. However, this mass lesion could not be determined with neither MG nor MR and could be realised only with USG and diagnosed to have cancer with biopsy.

Breast MR can functionally demonstrate the breast parencyma and the mass perfusion characteristics of the mass lesions at this base besides the morphological characteristics of the breast lesions such as; shape, contour and size [17]. In this research, radiological modalities such as Breast USG and MG were evaluated with CBE findings and additionally, in cases of BIRADS-3, BIRADS-4 and BIRADS-5, Breast MR was applied with the view of a radiologist and/or general surgery specialist. According to the MR results applied to $48(42 \%)$ of the patients defined as BIRADS-0 with MG, 10 (21\%) of the patients were diagnosed to have breast cancer. With this finding, MR is claimed to be a problem solving method in cases in which MG is restricted in defining (Table 3).

MR has become a commonly used monitoring method in differentiating between benign and malign in breast lesions. It has the highest specifity and sensitivity rate among the monitoring methods. It is indisputebly a useful method, especially in the cases where other monitoring methods are insufficient in providing required information. MR is definitely superior to other methods in determining the occurance of multifocal-multicentric cancer, with patients who need breast conserving surgery and evaluating scar tissue that occur after surgery $[18,19]$. In this research, multifocality occurance was determined with MR in a patient who was found to have BIRADS-5 single focus with MG and mastectomy was applied to this patient. USG defined BIRADS-5 lesions in two patients who had a breast reduction surgery and MG defined the lesions of these patients as BIRADS-0 and MR defined these as BIRADS-2 and the biopsy results were evaluated as benign.

According to the guidelines published by American Cancer Association in 2007, MR applied in addition to mammography can determine the high rish breast cancer at early stages, especially in cases in which MG has low sensitivity [20]. Breast MR is the most sensitive method in determining the breast cancer. Especially the conventional monitoring methods cannot easily differentiate between malign and benign cases. In such cases Breast MR is used as the problem solving method. Cases that are suspected to be malign and classified as BIRADS 3 and 4 with USG and MG can be diagnosed with MR with a high sensitivity $[21,22]$. In the invasive breast tumors, MR can give the size closest to the pathological size [23]. In this research Breast MR was done to 5 patients who were defined as BIRADS-4 with MG, and two of them were defined to have BIRADS-5 and the biopsy results revealed breast cancer and three of the patients were found to have BIRADS-2, 3 in Breast MR. The biopsies of these three patients were found to be benign.

Breast MR has a high sensitivity rate $(83 \%-100 \%)$, but the specifitiy rate of it is low $(23 \%-80 \%)$. MR should be used, when USG and MG are inefficient in defining the breast mass lesions [24]. As the specifity rate of MR is low, biopsy procedures to be carried as the result of MR will not always be sufficient in diagnosing 
the cancer. In this research, $35(31 \%)$ of the patients, to who biopsy was done because MR defined them as BIRADS-4 and BIRADS-5, were examined hispathologically and the results were benign.

Breast MR is inefficient in defining the breast insitu cancers and suspicious lesions under $3 \mathrm{mms}$ (14). In this research, breast insitu cancer could not be defined with $\mathrm{MR}$ in one of the patients and it was defined with MG. One of the patients was defined as BIRADS-5 with USG and the cancer found in the biopsy was not determined in MR (Table 4).

Cor biopsy is a more widely used method in breast cancer diagnosis. It has many advantages such as; acquiring adequate tissue sample, diagnosing fast, allowing receptor use and being cheaper than the open biopsy. The implementation of the procedure accompanied with USG decreases the false negativity rate to $0.2 \%$. This application allows breast conserving surgery and sentinel lymph node mapping while open biopsy doesn't, because the lymphatics remain unharmed $[25,26]$. In this research, all the 112 biopsies were done accompanied with USG and 27 (24\%) of the patients were diagnosd to have cancer. All of these patients were surgically operated.

\section{Conclusion}

USG and MR are prominent monitoring methods evaluating the breast diseases. Breast MR is a monitoring method that can be used in addition to USG and MG in suitable indications. Breast MR has a sensitivity rate close to MG. It is a problem solving method with cases of BIRADS-0 and BIRADS-4 breast lesions, where MG is inefficient.

\section{REFERENCES}

[1] A. A. Hatipoğlu and A. M. Tuncer, "Türkiyede Kanser Kontrolü 1 (Baski)," Onur Matbacılık, Ankara, 2007.

[2] J. W. Leung, "Screening Mammography Reduced Morbidity of Breast Cancer Treatment," American Journal of Roentgenology, Vol. 184, 2005, pp. 1508-1509.

[3] R. A. Denise, et al., "Imaging and Cancer: Research Strategy of the American College of Radiology İmaging Network," Radiology, Vol. 235, 2005, pp. 741-751. doi:10.1148/radiol.2353041760

[4] A. M. Tuncer and T. C. Sağlık, "Bakanlığı Kanserle Savaş Dairesi Başkanlığı, Kadınlarda Meme Kanseri Taramaları İçin Ulusal Standartlari,” 2004. http://www.ukdk.org/pdf/meme_standart.pdf

[5] A. S. Majid, et al., "Missed Breast Carcinoma: Pitfalls and Pearls," Radiographics, Vol. 23, 2003, pp. 881-895. doi:10.1148/rg.234025083

[6] M. Mahesh, "AAPM/RSNA Physics Tutorial for Residents: Digital Mammography: An Overview," Radiographics, Vol. 24, 2004, pp. 1747-1760. doi:10.1148/rg.246045102

[7] L. E. Duijm, et al., "Value of Breast İmaging in Women with Painful Breasts: Observational Follow Up Study," British Medical Journal, Vol. 317, 1998, pp. 1492-1495. doi:10.1136/bmj.317.7171.1492

[8] T. M. Kolb, et al., "Comparison of the Performance of Screening Mammography, Physical Examination, and Breast US and Evaluation of Factors That İnfluence Them: An Analysis of 27, 825 Patient Evaluations," Radiology, Vol. 225, 2002, pp. 165-175. doi:10.1148/radiol.2251011667

[9] M. C. Segel, et al., "Advanced Primary Breast Cancer: Assessment Mammography of Response to İnduction Chemotherapy," Radiology, Vol. 169, 1988, pp. 49-54.

[10] T. Rezanko, "Triple Test and Algorithm in Diagnosis of Breast Tumors," Journal of Breast Health, Vol. 3, 2008, pp. 143-150.

[11] M. Sant, et al., "Time Trends of Breast Cancer Survival in Europe in Relation to İncidence and Mortality," International Journal of Cancer, Vol. 119, No. 10, 2006, pp. 2417-2422. doi:10.1002/ijc.22160

[12] V. Özmen, "Dünya'da ve Türkiye'de Meme Kanseri Tarama (Screening) ve Kayıt Programlari," Journal of Breast Health, Vol. 2, 2006, pp. 55-58.

[13] S. W. Duffy, et al., "The Swedish Two-County Trial of Mammographic Screening: Cluster Randomisation and End Point Evaluation," Annals of Oncology, Vol. 14, No. 8, 2003, pp. 1196-1198. doi:10.1093/annonc/mdg322

[14] P. Boyle, "Recommendation for Mammografhic Screening after the Dust Settles," 8th International Conference: Primary Therapy of Early Breast Canser SL, 12-15 March 2002.

[15] R. Ballard-Barbash, et al., "Exploring the Role of Prevention, Screening and Treatment in Canser Trends in Perry ML," In: American Society of Clinical Oncology: Educational Book, 2002, pp. 127-136.

[16] R. Doğan, et al., "Follow-Up Protocolof with Negative Findings or Non-Palpabl Benign Breast Lesion: Mamographic and Ultrasonographic BI-RADS Assessment and Ultrasonography Guided Fine Needle Aspiration Biopsy," Journal of Breast Health, Vol. 3, 2007, pp. 58-62.

[17] C. Kuhl, "The Current Status of Breast MR İmaging. Part I. Choice of Technique, İmage İnterpretation, Diagnostic Accuracy, and Transfer to Clinical Practice," Radiology, Vol. 244, 2007, pp. 356-378. doi:10.1148/radiol.2442051620

[18] S. H. Heywang-Korunner, et al., "Diagnostic İmaging," 2nd Edition, Thineme, Ludwisburg, 2001.

[19] S. G. Orel, "MR İmaging of the Breast," Radiologic Clinics of North America, Vol. 38, No. 4, 2000, pp. 899913. doi:10.1016/S0033-8389(05)70208-6

[20] D. Saslow, et al., "American Cancer Society Breast Cancer Advisory Group. American Cancer Society Guidelines for Breast Screening with MRI as an Adjunct to Mammography," A Cancer Journal for Clinicians, Vol. 57, No. 2, 2007, pp. 75-89. doi:10.3322/canjclin.57.2.75

[21] W. A. Berg, et al., "Diagnostic Accuracy of Mammography, Clinical Examination, US, and MR İmaging in Preoperative Assessment of Breast Cancer," Radiology, Vol. 233, 2004, pp. 830-849. 
doi:10.1148/radiol.2333031484

[22] D. A. Bluemke, et al., "Magnetic Resonance İmaging of the Breast Prior to Biopsy," The Journal of the American Medical Association, Vol. 292, No. 22, 2004, pp. 27352742. doi:10.1001/jama.292.22.2735

[23] M. Van Goethem, et al., "Magnetic Resonance İmaging in Breast Cancer," European Journal of Surgical Oncology, Vol. 32, 2006, pp. 901-910. doi:10.1016/j.ejso.2006.06.009

[24] I. Başara, et al., "Diagnostıc Values of Mamography,
Ultrasonography and Dynamic Enhanced Magnetic Resonance İmaging in Breast Lesions," Journal of Breast Health, Vol. 7, 2011, pp. 118-126.

[25] M. F. Ernst and J. A. Roukema, "Diagnosis of NonPalpable Breast Cancer: A Review," Breast, Vol. 11, No. 1, 2002, pp. 13-22. doi:10.1054/brst.2001.0403

[26] S. D. Lind, et al., "Stereotactic Core Biopsy Reduces the Reexcision Rate and the Cost of Mammographically Detected Cancer," Journal of Surgical Research, Vol. 78, No. 1, 1998, pp. 23-26. doi:10.1006/jsre.1998.5380 